\title{
DIAMOND CUTTING ACCELERATED BY AN ELECTRIC ARC
}

\author{
By Chauncey G. Peters, Karl F. Nefflen, and Forest K. Harris
}

\begin{abstract}
The method universally employed for cutting plane surfaces or facets on diamonds has been to place the diamond in contact with a flat cast-iron lap charged with diamond powder and rotated at about 2,000 revolutions per minute. In the work described in this paper it was found that by producing a high-voltage electric arc at the contact between the diamond and the lap, the cutting rate is materially increased for all orientations of the diamond, and good progress can be made directly on a natural octahedron face, where cutting without the arc is almost impossible.

By applying the arc to a diamond saw the sawing rate is greatly increased, and diamonds can be sawed regardless of the orientation of the cut relative to the crystal axes.
\end{abstract}

\section{CONTENTS}

I. Introduction 1 Page

II. Method and apparatus.

III. Preparation of the lap

IV. Control of cutting rate 589

1. Diamond powder

2. Mixing oil

3. Lap speed $\ldots$

4. Electric conditions

V. Test of cutting rate

VI. Test of sawing

VII. Summary

VIII. References

\section{INTRODUCTION}

In cutting surfaces on precision diamond tools required in research developments of standards of length, microhardness testing, methods for producing diamond dies, jewel bearings, etc., at the National Bureau of Standards, it was found that the rate of cutting was greatly increased by producing a high-voltage electric arc at the contact between the diamond and the surface of a rotating lap charged with diamond powder. The cutting method universally employed by the lapidaries since the beginning of the art $[1,2]$ has been to spin a flat cast-iron lap about 1 foot in diameter at approximately 2,000 revolutions per minute. Diamond powder mixed with olive oil or some other viscous material is fed onto the surface of the lap. The diamond is embedded in a wiping solder contained in a copper cup or dop, which in turn is supported by a wooden arm. The diamond is brought into 
contact with the surface of the spinning lap and cut until the desired surface, or facet, is produced.

Diamonds crystallize in the cubic system, which has three equal axes at right angles, in the form of octahedrons, dodecahedrons, and cubes $[1,3]$. It is well known that a facet can be most readily cut when it has certain orientations relative to the crystal axes. As a general rule, cutting is most rapid when the facet is parallel to one or two of the axes and the direction of motion of the lap surface is parallel to one of the axes. It is well established that cutting on an octahedron face, which is approximately at a 35 -degree angle to all three axes, is almost impossible.

The fact that under certain orientations the diamond is more resistant to abrasion than under others no doubt makes it possible to cut a diamond with its own powder. A part of the myriad of fine particles in the powder is always in the most resistant position and will abrade a surface oriented in a less resistant plane.

While making microhardness tests of diamonds from various sources, it was possible to make as many as 50 indentations, 30 microns long, in the flat surface of a diamond without injury to the point of a diamond indenter cut in the form of a four-sided pyramid. This led to the conclusion that the indenter point under compression had greater resistance to fracture than the diamond surface under tension. This may supply another explanation for the ability of powder particles to fracture or abrade the facet.

By applying the electric arc to diamond-cutting machines in general use, as described in this paper, good progress can be made when cutting is done in any direction directly on the octahedron face, and the rate of cutting on any surface can be closely controlled by adjusting the primary current.

\section{METHOD AND APPARATUS}

The diamond cutting apparatus developed at this Bureau is shown in figure 1 , and the wiring diagram is given in figure 2. The lap, $L$, of cast iron, copper, or other material supported by the base, $W$, is driven by the motor, $M$, at speeds ranging from 30 to 2,500 revolutions per minute. An arm, $B$, of insulating material such as 1-inch-square fiber bar, supports the diamond, $D$, and the weight, $F$.

The leads from the 110-volt, 60-cycle alternating-current outlet are connected to the primary terminals of a 5,000-volt, 50/1,300-voltampere power transformer, $T$. In one of the primary connecting leads a variable rheostat, $P$, of $400 \mathrm{ohms}$ is placed and in the other an alternating-current ammeter, $A$, of 2-ampere range. A capacitor, $C$, of about 0.001 to 0.008 microfarad is connected across the secondary leads of the transformer. The rheostat may be dispensed with if a current-limiting transformer, such as a 12,000-volt, 20-milliampere neon sign lighting transformer is used instead of the power transformer and a 0 to 120 -volt output Variac is placed between the alternatingcurrent outlet and the transformer.

One of the secondary leads of the transformer is connected to $F$ and the other to a brush, $E$, which makes electric contact with the bottom of lap, $L$, The diamond is mounted in a copper dop, high melting 


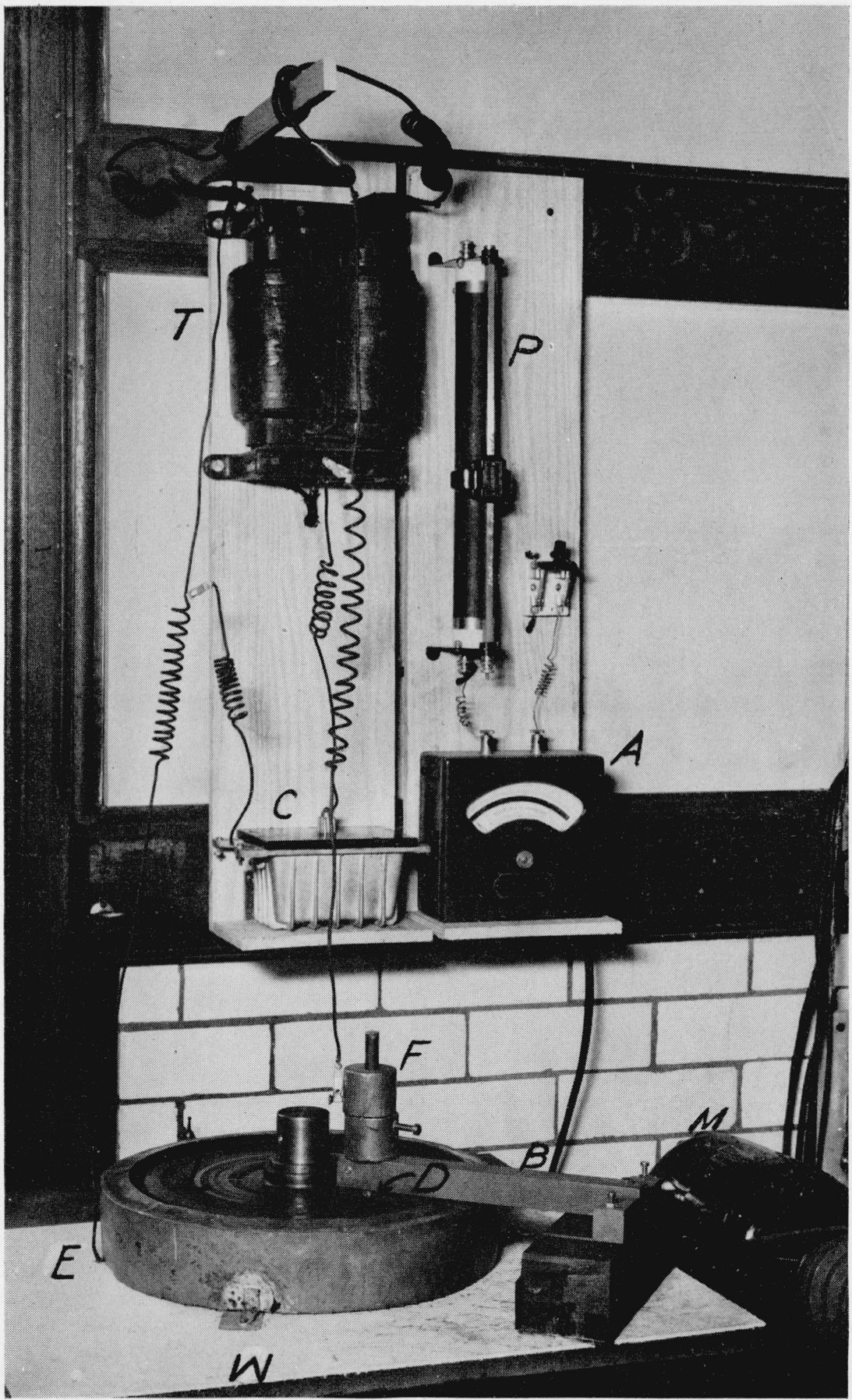

FIGURE 1.-Diamond lap with electric equipment and connections. 


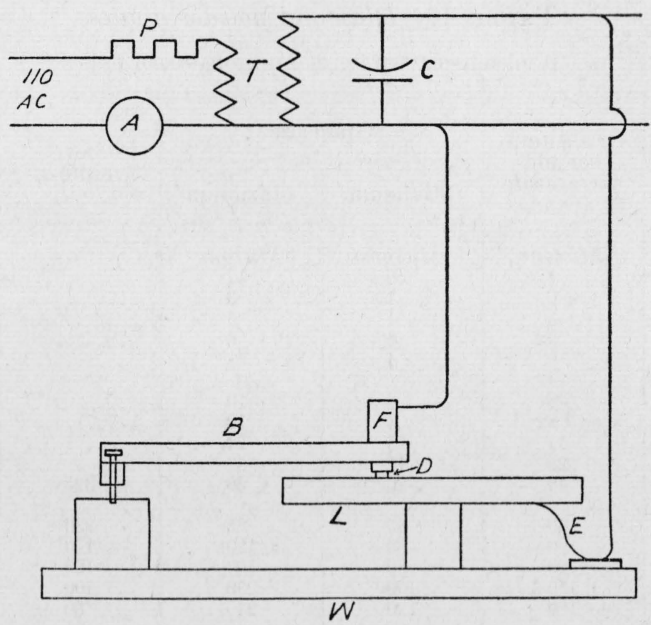

Figure 2.-Wiring diagram for apparatus shown in figure 1.

point solder being used, such as SAE No. E06, tin, lead, silver, or an alloy of equal weights of silver and aluminum and protrudes about $1 / 2$ to 1 millimeter from the surface of the surrounding solder. The heat conductivity of the copper reduces the chance of solder being melted by the frictional heat of the lap.

\section{PREPARATION OF THE LAP}

A cast-iron lap of high carbon and graphite content and containing numerous small pits or pockets is turned in a lathe on both sides and on the edge and carefully balanced. It was found that cross scoring of the top surface of the lap by hand or with a cupped wheel was not necessary, but the final cut should be light to produce a smooth surface. A small quantity of diamond powder and a few drops of oil are applied to the lap, mixed well and rubbed into the surface with a flat, hardened steel block. A mixture of oil and powder can be applied while the lap is in motion, but this is rather wasteful of powder.

\section{CONTROL OF CUTTING RATE}

\section{DIAMOND POWDER}

In a recent report on diamond powder prepared by the Nationa Bureau of Standards for the War Production Board, N. R. C. 535' Serial No. W-144, grade specifications for diamond powder were, proposed. With the cooperation of the diamond industry, the Trade Standards Division of this Bureau has prepared the following specifications for standard grades, which when adopted should result in uniform grade designation.

For diamond cutting, grades 25 and 40 were found to give good results. Grade 40 cut slightly faster than grade 25 , but the latter produced a better finish. 
Table 1.-Diamond powder grades

[ 1 micron $=0.00004$ in.; 25.4 microns $=0.001$ in.]

\begin{tabular}{|c|c|c|c|}
\hline \multirow{2}{*}{$\begin{array}{l}\text { Grade num- } \\
\text { ber and } \\
\text { average size }\end{array}$} & \multicolumn{2}{|c|}{ Grain size } & \multirow{2}{*}{$\begin{array}{c}\text { Sieve } \\
\text { number }\end{array}$} \\
\hline & Minimum & Maximum & \\
\hline $\begin{array}{c}\text { Microns } \\
1 \\
2 \\
3 \\
3 \mathrm{x} \\
6\end{array}$ & $\begin{array}{c}\text { Microns } \\
0 \\
1 \\
1 \\
0 \\
4\end{array}$ & $\begin{array}{c}\text { Microns } \\
2 \\
3 \\
5 \\
6 \\
8\end{array}$ & - \\
\hline $\begin{array}{l}8 \\
8 \mathrm{x} \\
14 \\
25 \\
40\end{array}$ & $\begin{array}{r}6 \\
4 \\
8 \\
13 \\
20\end{array}$ & $\begin{array}{l}10 \\
12 \\
20 \\
37 \\
60\end{array}$ & 325 \\
\hline $\begin{array}{r}60 \\
90 \\
120 \\
150 \\
180\end{array}$ & $\begin{array}{r}35 \\
60 \\
80 \\
100 \\
120\end{array}$ & $\begin{array}{r}85 \\
120 \\
160 \\
200 \\
240\end{array}$ & $\begin{array}{r}230 \\
170 \\
120 \\
100 \\
80\end{array}$ \\
\hline $\begin{array}{l}250 \\
400\end{array}$ & $\begin{array}{l}150 \\
250\end{array}$ & $\begin{array}{l}350 \\
550\end{array}$ & $\begin{array}{l}60 \\
40\end{array}$ \\
\hline
\end{tabular}

\section{MIXING OIL}

Experiments were run, a number of different oils being used for mixing with the diamond powder. Vegetable oils such as olive oil and also asphalt-base mineral oils burned off rapidly from the heat of the arc. The best results were obtained with paraffine base, high flash point Pennsylvania oils of 30 to $50 \mathrm{SAE}$ viscosity number.

\section{LAP SPEED}

The lap was revolved at various speeds between 30 and 2,500 revolutions per minute. At the lower speeds the cutting rate was almost as fast as at the higher speeds, but the surface of the diamond showed a ground appearance. Speeds near 2,000 revolutions per minute gave good cutting rate and surface finish. At the low speeds the dop remained cool; therefore, the heat of the arc, although sufficient to account for most of the burning away of the oils at the contact surface, had negligible effect on the dop and solder. Almost all the heat transmitted to those elements must be produced by the friction between the diamond and high speed lap.

\section{ELECTRIC CONDITIONS}

Returning to figures 1 and 2 , the maximum cutting rate was attained with an applied voltage of 110 volts, a current of about 0.5 ampere in the primary of 50/1 transformer, $T$, and a capacitance across the secondary leads of 0.005 to 0.008 microfarad. To obtain that current in the primary circuit the rheostat, $P$, was adjusted to about 200 ohms. To decrease the cutting rate, the primary current and the capacitance were reduced.

Increased current augmented the arc, which in turn produced increased burning of the mixing oil and a slower cutting rate. 


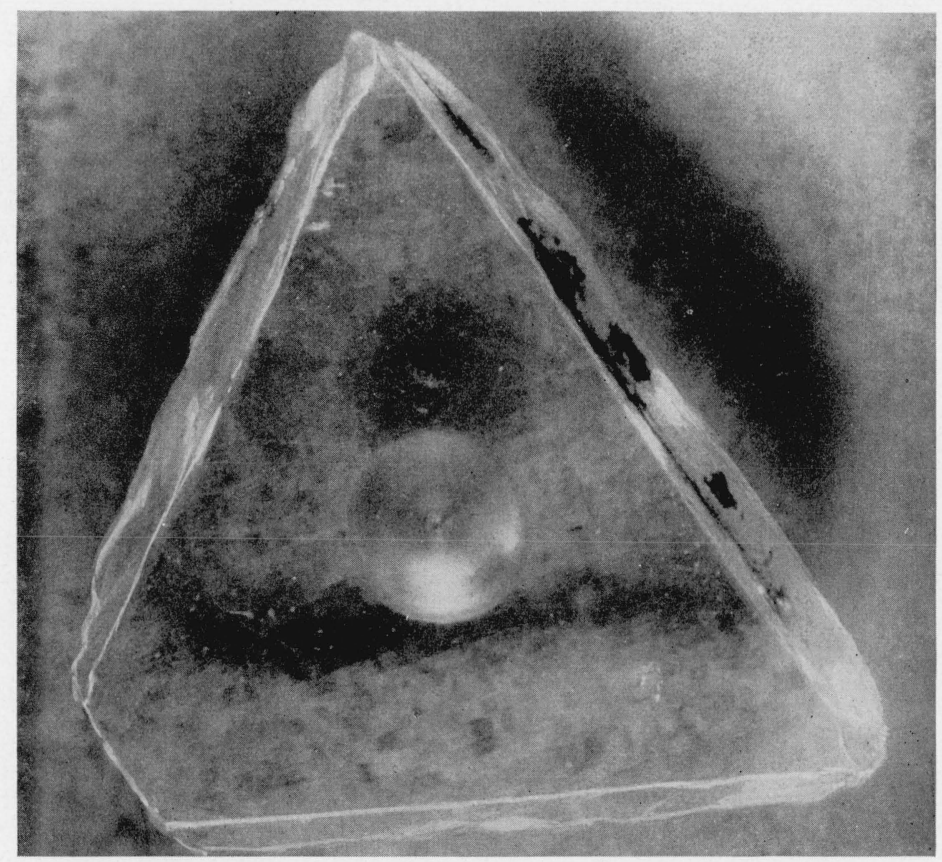

Figure 3.-Facet cut on the octahedron face of a diamond. 


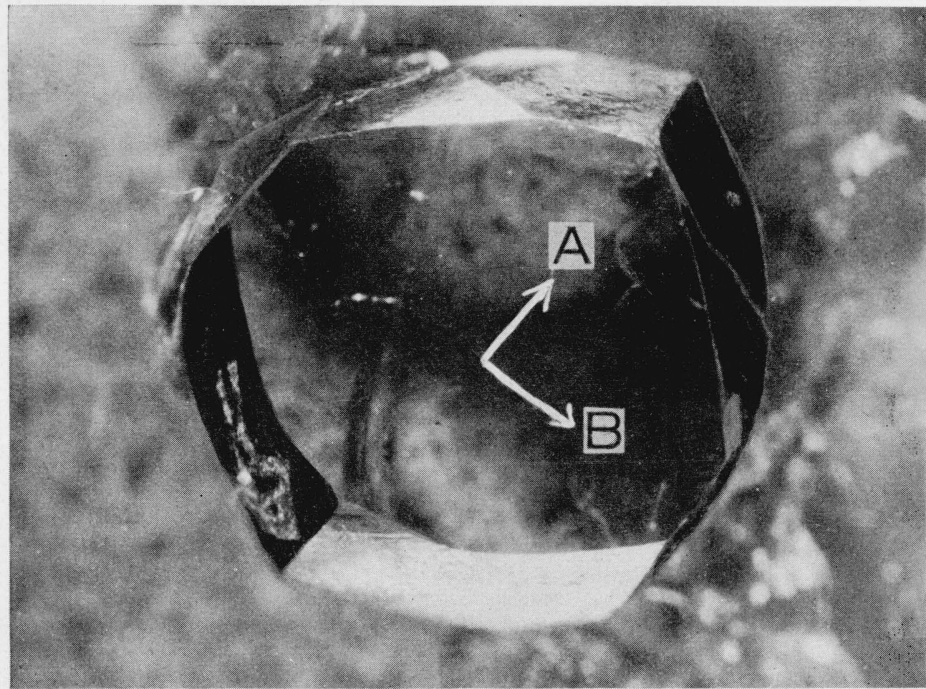

Figure 4.-Facet cut on the dodecahedron face of a diamond. Direction $A$, parallel to one axis; direction $B$, perpendicular to one axis.

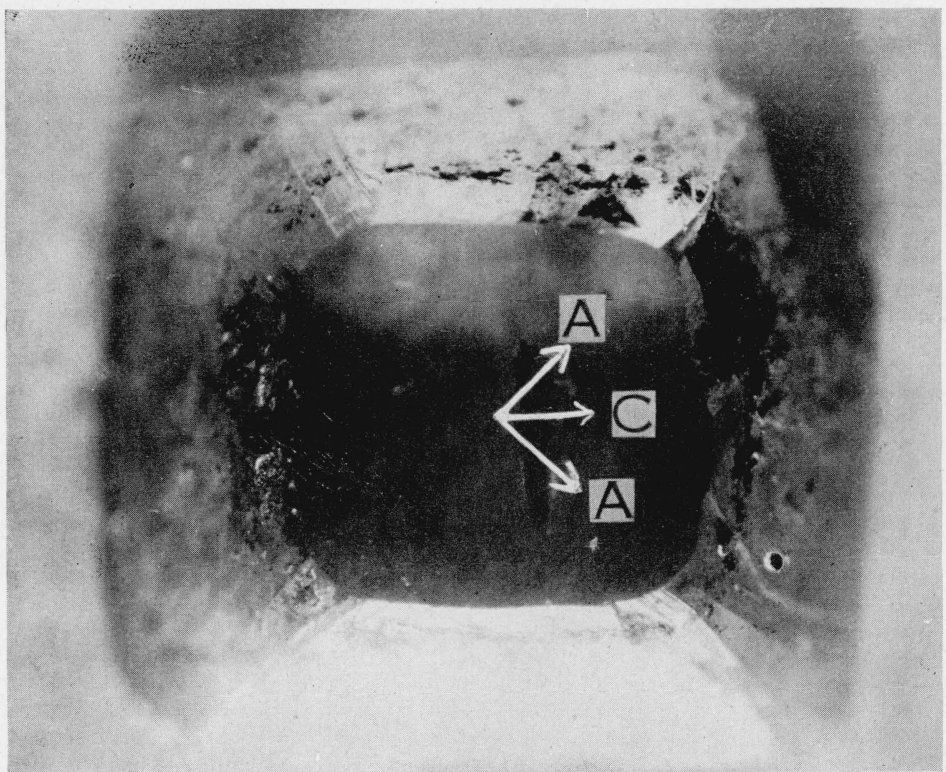

Figure 5.-Facet cut on cube face of a diamond.

Direction $A$, parallel to one axis; direction $C, 45$ degrees to two axes. 


\section{TEST OF CUTTING RATE}

To determine the effect of the electric are on the cutting rate, experiments were run on some 25 different diamond crystals, including octahedrons, cubes, dodecahedrons, three-point, capes and macles.

Several well-formed diamond crystals were especially prepared by cutting a base and table accurately parallel to one another. The distances between these two facets before and after making the test cuts were carefully measured with a micrometer. The tables were cut to an area of about 8 square millimeters, 0.013 square inch, before the rate data were recorded. Thereafter, the time required to remove an additional thickness of from 0.010 inch to 0.040 inch was used to determine the cutting rate, which is taken as the time required to remove a layer of 0.001 -inch thickness from a facet of about 8 square millimeters, 0.013-square-inch area. Test cuts were also made on the back faces of diamond dies, figure 3 . The thickness removed was accurately determined by means of a micrometer microscope sighted through the die window.

The base and table in each case were cut accurately parallel to one of the principal crystal faces of the diamond. Figure 3 shows the facets cut parallel to the octahedron face, which makes angles of 35 degrees with the three crystal axes. Figure 4 shows the facets cut parallel to a dodecahedron face, which is parallel to one axis and makes angles of 45 degrees with the other two axes. Figure 5 shows the facets cut parallel to the cube face, which is parallel to two axes and perpendicular to the third.

On the octahedron face the rate of cutting was the same for all directions of motion of the lap surface. On the dodecahedron face the maximum cutting rate resulted when the direction of the lap motion was parallel to the crystal axis shown by $A$ in figure 4 , and the rate was at a minumum at 90 degrees to that direction as shown by $B$. For the cube face, the cutting rate was at a maximum when the lap direction was parallel to one of the two axes as shown by $A$ in figure 5 and at a minimum at 45 degrees to those directions as represented by $C$. For intermediate directions the cutting rate lies between these extremes. In table 2 are given the maximum and minimum cutting rates, as defined above, with and without the arc, for diamonds cut on the three crystal faces. For like conditions of crystals face and lap direction there was but little variation of rate between individual diamonds.

TABLE 2.-Comparison of cutting rates

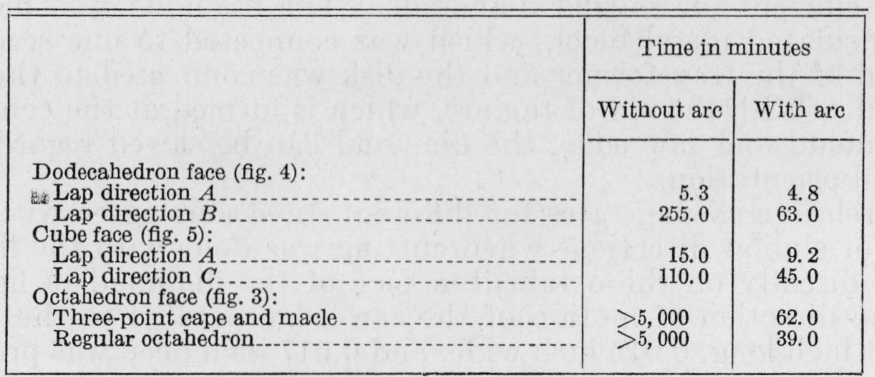


From these data it is seen that when cutting is done on the dodecahedron face with the lap direction parallel to the axis, $A$, (which appears to be the most favorable condition for cutting diamonds), the rate is about the same with or without the arc. With the lap direction at right angles to the axis, $B$, however, the arc increases the cutting rate about four times.

On the cube face, when the cutting was parallel to one axis and at right angles to the other, $A$, figure 5 , the arc doubled the rate, and at 45 degrees to the two axes, $C$, it increased the rate by about two and one-half times.

For the octahedron faces without the arc, no cutting resulted in any direction of the lap motion; that is, not as much as 0.0001 inch layer could be removed in a day's time. This agrees with the experience of expert lapidaries, as recorded in the literature [2,4].

With the arc, however, the cutting rate on the octahedron face equals the rates found for the unfavorable directions on the cube and dodecahedron faces, which in turn have been augmented about four times by the use of the arc. That the arc facilitates such rapid cutting directly on the octahedron face is the most striking result of this work. This should make it possible, in designing industrial diamond tools, to take full advantage of the orientation of the crystallographic directions of the diamond with respect to the cutting surface of the tool [5].

The data presented here have been obtained from facets cut parallel to the three principal crystal faces. Additional quantitative cuttingrate data are being accumulated for other definite orientations of the facets.

\section{TEST OF SAWING}

Sawing diamonds by established methods can only be performed in certain directions parallel to either a cube or dodecahedron face and have been called the "sawing grain." According to Grodzinsky, "It is almost impossible to saw the diamond in other directions even if they differ a few degrees from the original axis" [2, p. 44]. It should be added that when sawing parallel to the cube or dodecahedron face the direction of motion of the saw edge should be nearly parallel to one of the crystal axes.

To test the effect of the arc when applied to diamond sawing, a saw previously employed for slicing sapphire and spinel during an investigation of jewel bearing materials was employed. This machine, which has a phosphor bronze disk 6 inches in diameter and 0.012 inch thick rotating at about 2,800 revolutions per minute, cannot be considered efficient for sawing diamonds. The diamond was mounted in an insulated metal block, which was connected to one secondary terminal of the transformer and the disk was connected to the other terminal. With the aid of the arc, which is formed at the contact of the diamond and saw edge, the diamond can be sawed regardless of the axial orientation.

The relative sawing rates for different directions agree with those found for similar directions when cutting was done with the flat lap. Sawing directly on the octahedron face of the diamond in figure 3, with the direction of motion of the saw edge parallel to the face, a cut 0.14 inch long, 0.015 inch wide, and 0.017 inch deep was produced in 75 minutes. Sawing on the dodecahedron face of the diamond 
in figure 4 with the direction of motion of the edge of the saw parallel to the crystal axis, that is, in direction $\mathrm{A}$, a cut 0.14 inch long, 0.020 inch wide, and 0.030 inch deep was made in 15 minutes.

When sawing at right angles to the crystal axis or in direction B, figure 4, the sawing rate was about the same as for the octahedron face.

If we define the cutting or sawing direction as the direction of motion of the diamond particles imbedded in the lap or the edge of the saw, the maximum cutting rate results when that cutting direction is parallel to one of the crystal axes. Under this condition the cutting direction makes angles of from about 35 to 45 degrees with the octahedron faces or clevage planes. When the cutting direction is parallel to the octahedron faces or clevage planes the cutting rate is at a minimum. For other orientation the cutting, or sawing, rate lies between these two extremes. These same rules should hold for the rate of wear in diamond dies.

Experiments are now in progress the purpose of which is to find the explanation of the electric phenomena involved in the arc cutting, sawing, and drilling of diamonds. However, the data now accumulated do not justify definite conclusions regarding the nature of the process.

\section{SUMMARY}

By connecting the lap of a diamond cutting machine to one of the secondary terminals of a 5,000-volt power transformer having a current of about 0.5 ampere flowing through the primary circuit and the dop to the other terminal, a bluish arc is produced at the contact of the diamond and lap. Under these conditions the cutting rate is materially increased for all orientations of the facets relative to the crystal axes of the diamond. Good progress can be made directly on the octahedron faces, where no appreciable cutting could be effected by the methods generally employed. Similarly, by applying the arc to the diamond saw the sawing rate is greatly increased and cuts can be made in the diamond under any orientation.

\section{REFERENCES}

[1] Kraus and Slawson, Gems and gem materials, fourth edition (McGraw-Hill Book Co., Inc., New York, N. Y. and London, 1941).

[2] Paul Grodzinski, Diamonds and gem stones-industrial production, first edition (N. A. G. Press, Ltd., London, and Henry Paulson \& Co., 37 S. Wabash Ave., Chicago, Ill., 1942).

[3] Dana's textbook of mineralogy, p. 70, fourth edition (John Wiley \& Sons, Inc., New York, N. Y. and Chapman \& Hall, Ltd., London, 1932).

[4] Kraus and Slawson, American Mineral. 24, 661 (November 1939).

[51 Kraus and Slawson, American Mineral. 26, 153 (1941).

Washington, March 30, 1945. 